\title{
Landing Footprint Computation for Entry Vehicles
}

\author{
Amitabh Saraf, ${ }^{*}$ James A. Leavitt, ${ }^{\dagger}$ and Kenneth D. Mease ${ }^{\ddagger}$ \\ University of California, Irvine, CA 92697 \\ Mark Ferch ${ }^{\S}$ \\ University of Stuttgart, 70550 Stuttgart, Germany
}

\begin{abstract}
A method is developed for generating landing footprints for entry vehicles in near realtime, as needed for an on-board flight management system. The method described in the paper is built around a previously developed, acceleration-based trajectory planner. The boundary of the footprint is constructed from the endpoints of extreme downrange or crossrange trajectories generated by the planner. The strengths of the method include fast computation time, respecting path constraints such as vehicle heating limits, accounting for Coriolis effects and constructing angle of attack profiles that are nearly optimal for long range glide. The accuracy of the landing footprint generator is determined by direct comparison with footprints computed with a general purpose optimization program.
\end{abstract}

\section{Introduction}

An important capability for the safe operation of an entry vehicle is to predict, from any initial position and velocity, the landing footprint, i.e., the set of feasible landing sites. A landing site is feasible if the vehicle has sufficient performance to reach it without violating any safety constraints concerning the vehicle or crew. The landing footprint provides critical information for mission planning in both nominal and abort situations. For autonomous vehicle operation, footprint calculation should be fast and reliable, so that it can be executed on board in a timely manner.

To calculate the footprint, the boundary points of the landing footprint should be determined. However, the boundary points are the end points of optimal trajectories, and solving a family of optimal control problems rapidly and reliably is a daunting task. Thus the challenge in developing an algorithm to calculate the landing footprint is to find a method that simplifies the calculations, yet generates a sufficiently close approximation to the actual landing footprint. Features that make this task difficult, yet are too influential to be neglected, are the constraints on heating, acceleration and dynamic pressure, and the Coriolis terms in the entry dynamics. The Coriolis terms, i.e., the effects of earth rotation, introduce significant asymmetry in the landing footprint.

Previous work has been done in the area of landing footprint generation. Vinh ${ }^{1}$ provides a method for producing optimal landing footprints in an idealized setting with no vehicle constraints or Coriolis effects. Ngo and Doman ${ }^{2}$ develop a method for generating near-optimal landing footprints, that is based on the landing footprint work of Vinh, and also does not account for Coriolis effects or vehicle constraints. The glided flight portion ${ }^{3}$ of the Shuttle Abort Flight Manager (SAFM) generates landing footprints in a manner that is similar to the method presented in this paper, using simulation runs with constant bank direction to generate the side boundaries of the footprint, and upper and lower drag constraint boundaries to determine the far and near edges of the footprint.

\footnotetext{
*Postdoctoral Researcher, Department of Mechanical and Aerospace Engineering, asaraf@uci.edu, Member AIAA, current address: Centre for AI and Robotics, Bangalore, India

${ }^{\dagger}$ Graduate Research Assistant, Department of Mechanical and Aerospace Engineering, jleavitt@uci.edu

¥Professor, Department of Mechanical and Aerospace Engineering, kmease@uci.edu, Associate Fellow AIAA

$\S$ Visiting Student, Institute of Flight Mechanics and Control, mferch@uci.edu
} 
The computational method presented here is built around the planning function in Evolved Acceleration Guidance Logic for Entry (EAGLE), ${ }^{4}$ though the landing footprint research documented in this paper has led to improvements in EAGLE which, before this paper, have not been published. The EAGLE planner designs feasible trajectories, accounting for vehicle constraints and Coriolis effects. The algorithm computes a landing footprint for an entry condition determined by the following flight parameters: velocity, altitude, flight path angle, heading, latitude and longitude. The end of entry phase is determined by a desired final velocity. This is consistent with the space shuttle operation in which the entry phase is followed by the Terminal Area Energy Management (TAEM) phase. TAEM phase begins when the shuttle slows down to a specific velocity and it is desired that at that instant, the vehicle altitude, latitude, longitude and heading should be within prescribed limits of the corresponding desirable values. The footprint calculation algorithm determines all possible latitude and longitude points that can be reached as the vehicle reaches the TAEM velocity and TAEM altitude. The final heading angle is not an important requirement to construct the footprint because of the flexibility in the choice of final heading. According to NASA's Advanced Guidance and Control (AGC) study regulations, the only requirement for the final heading is that at the end of entry phase, the vehicle should be pointing towards the Heading Alignment Cone (HAC) point. ${ }^{5}$ EAGLE uses this freedom by moving the TAEM target point along a $30 \mathrm{nmi}$ TAEM circle to eliminate the heading error to the HAC point. ${ }^{4}$

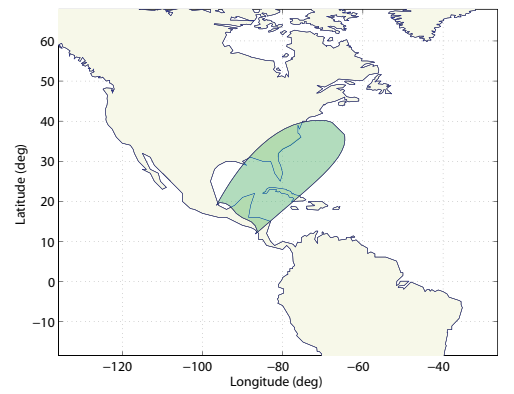

Figure 1. Sample landing footprint

We do not consider trajectories that skip out of the atmosphere to achieve greater range. Such trajectories can enlarge the footprint and thus may be important, but they are not considered here.

\section{A. Entry Dynamics}

The translational dynamics of an atmospheric entry vehicle are defined with respect to a planet-fixed coordinate frame. Since the vehicle during entry is unpowered, the total energy of the vehicle, determined by $E=V^{2} / 2-\mu / r$, where $V$ is the planet-relative velocity of the vehicle, $r$ is the radial distance from the vehicle's center of mass to the planet center, and $\mu$ is the gravitational constant, monotonically decreases along the trajectory. Energy is an appropriate independent variable for the dynamics, ${ }^{6-8}$ since there is no concern about the time that entry begins or ends and the target final conditions are specified at either a final velocity or final energy. With energy as the independent variable, the vehicle's translational motion can be modelled by five state equations. The control variables are bank angle $(\sigma)$ and angle of attack $(\alpha)$. Angle of attack appears in the equations of motion through lift and drag, while bank angle appears explicitly. Neglecting winds and centripetal acceleration from planet rotation, the equations of motion, consistent with those given in Ref. 1 except for the transformation to energy as the independent variable, are

$$
\begin{aligned}
\theta^{\prime} & =-\frac{\cos \gamma \cos \psi}{r \cos \phi}\left(\frac{1}{D}\right) \\
\phi^{\prime} & =-\frac{\cos \gamma \sin \psi}{r}\left(\frac{1}{D}\right) \\
r^{\prime} & =-\sin \gamma\left(\frac{1}{D}\right) \\
\psi^{\prime} & =\frac{\cos \psi \tan \phi \cos \gamma}{r}\left(\frac{1}{D}\right)+\frac{1}{V^{2} \cos \gamma}\left(\frac{L \sin \sigma}{D}\right)+\mathcal{C}_{\psi} \\
\gamma^{\prime} & =\left(g-\frac{V^{2}}{r}\right) \frac{\cos \gamma}{V^{2}}\left(\frac{1}{D}\right)-\frac{1}{V^{2}}\left(\frac{L}{D} \cos \sigma\right)+\mathcal{C}_{\gamma}
\end{aligned}
$$

where $\theta$ is the longitude, $\phi$ is the latitude, $\psi$ is the heading angle with $\psi=0$ as due east, and $\gamma$ is the flight path angle. Both $\gamma$ and $\psi$ describe the orientation of the planet-relative velocity vector. The bank angle $\sigma$ is defined such that a bank to the right is positive and zero bank corresponds to the lift vector directed 
upward in the longitudinal plane. The lift $L$ and drag $D$ accelerations are given by

$$
\begin{aligned}
L & =\frac{1}{2} \rho(r) V^{2} \cdot \frac{S}{m} \cdot C_{L}(\alpha, M) \\
D & =\frac{1}{2} \rho(r) V^{2} \cdot \frac{S}{m} \cdot C_{D}(\alpha, M)
\end{aligned}
$$

where $\rho(r)$ is the density as a function of altitude, $C_{L}(\alpha, M)$ and $C_{D}(\alpha, M)$ are the lift and drag coefficients as functions of angle of attack $\alpha$ and Mach number $M, S$ is the reference area, and $m$ is the vehicle mass. Gravity is modeled as $g=\mu / r^{2}$. The density variation with altitude is modeled using the following exponential equation

$$
\rho(r)=\rho_{0} e^{-\frac{r-r_{0}}{h_{s}}}
$$

where $r_{0}$ is the equatorial radius of the planet, and $h_{s}$ (called scale height) and $\rho_{0}$ are constants. The terms $\mathcal{C}_{\psi}$ and $\mathcal{C}_{\gamma}$ account for the contributions of the Coriolis acceleration due to planet rotation. These terms are given as

$$
\begin{aligned}
& \mathcal{C}_{\psi}=-\left(\frac{2 \omega_{p}}{V D}\right)(\tan \gamma \sin \psi \cos \phi-\sin \phi) \\
& \mathcal{C}_{\gamma}=-\left(\frac{2 \omega_{p}}{V D}\right) \cos \psi \cos \phi
\end{aligned}
$$

where $\omega_{p}$ is the angular rate of planet rotation.

\section{B. Path constraints}

The vehicle has maximum limits for dynamic pressure, aerodynamic acceleration, and heating rate. The limit on dynamic pressure is given by

$$
Q=\frac{1}{2} \rho V^{2} \leq Q_{\max }
$$

The constraint on aerodynamic acceleration is expressed as the following constraint on normal acceleration

$$
L_{n}=L \cos \alpha+D \sin \alpha \leq A_{\max }
$$

The heating rate must also not exceed the design limits of the vehicle's thermal protection. Thus, the heating rate is constrained according to the heating model

$$
\dot{q}=c \rho^{1 / 2} V^{3.15} \leq \dot{q}_{\max }
$$

where $c$ is a constant that is dependent on the heating model.

\section{Algorithm Description}

The algorithm to construct the footprint is presented in two sections. The first section discusses the construction of a footprint with a fixed angle of attack profile. This capability is applicable either to a vehicle for which the trim angle of attack is not adjustable or to a vehicle for which one wants to estimate conservatively the footprint with a nominal angle of attack profile. The second section incorporates a procedure for adjusting the angle of attack profile for maximum range into the footprint generator. This capability is especially useful for abort planning when it is critical to know the maximum achievable footprint.

\section{A. Footprint for fixed alpha profile}

In this section we assume that the angle of attack profile is a prescribed function of normalized energy. We define normalized energy as $\tilde{E}=\left(E-E_{i}\right) /\left(E_{f}-E_{i}\right)$, where $E$ is the current energy and $E_{i}$ and $E_{f}$ are the initial and the desired final energy values respectively. With this definition $\tilde{E}=0$ at the start of the entry phase and $\tilde{E}=1$ at the nominal TAEM condition.

The algorithm generates the boundary of the landing footprint. The footprint boundary is approximated as a polygon whose vertices are endpoints of flyable tractories created by the algorithm. "Flyable" in this context is taken to mean "originating from control profiles that are continuous up to the second time 
derivative and that observe vehicle limits on value, rate, and angular acceleration". Trajectories are created by first designing drag versus energy profiles and scheduling the bank direction with energy. Drag is related to bank angle by the equation

$$
\frac{L}{D} \cos \sigma=\frac{1}{b}\left(D^{\prime \prime}-a\right)
$$

where

$$
\begin{aligned}
a & =D\left(\frac{C_{D}^{\prime \prime}}{C_{D}}-\frac{C_{D}^{\prime 2}}{C_{D}^{2}}\right)+D^{\prime}\left(\frac{C_{D}^{\prime}}{C_{D}}+\frac{2}{V^{2}}\right)-\frac{4 D}{V^{4}}+\frac{1}{D V^{2}}\left(\frac{1}{h_{s}}+\frac{2 g}{V^{2}}\right)\left(g-\frac{V^{2}}{r}\right)+\left(\frac{1}{h_{s}}+\frac{2 g}{V^{2}}\right) \mathcal{C}_{\gamma} \\
b & =-\frac{1}{V^{2}}\left(\frac{1}{h_{s}}+\frac{2 g}{V^{2}}\right)
\end{aligned}
$$

Using the nominal angle of attack profile (which determines the variation of $L / D$ with energy) and the planned drag profile, a reference bank angle magnitude profile is constructed as

$$
|\sigma|=\cos ^{-1}\left(\frac{D}{b L}\left(D^{\prime \prime}-a\right)\right)
$$

For flyable trajectories, bank sign can only change at points where $D(E)$ gives $|\sigma|=0$ per Eq. [10]. Otherwise, bank sign is independent of drag. Once the sign of the bank angle is chosen as a function of $E, \sigma(E)$ is known and the equations of motion (Eq. [1]) can be integrated numerically using $\alpha(E)$ and $\sigma(E)$ to obtain a trajectory. The distance traveled along a trajectory is related to drag and energy by

$$
S=-\int(1 / D) d E
$$

Therefore, by varying the drag profile it is possible to vary range. The polygon vertices for the left and right sides of the landing footprint are the endpoints of trajectories of increasing range. Since drag only determines the magnitude of bank angle, the same drag profiles are used to compute both sides. Left-side trajectories bank constantly to the left, while right-side trajectories always bank to the right. The near edge of the landing footprint corresponds to the highest drag profile that can be flown without violating path constraints. The variation in crossrange along the near edge comes from varying the initial bank sign and the time at which a single bank sign reversal maneuver is performed. The far side of the landing footprint is similar to the near side, except a minimum drag profile is used. The minimum drag profile is taken as the drag that results from using bank angle to track a constant zero flight path angle. An example of such a landing footprint is given in Fig. 1.

The flyable upper drag boundary is computed using the following steps. The three path constraints defined in the previous section are converted to limits on drag for the fixed angle of attack profile. The dynamic pressure constraint takes the form

$$
D<Q_{\max } S C_{D}
$$

For the normal acceleration limit, we have

$$
D<\frac{A_{\max }}{\sin \alpha+\frac{L}{D} \cos \alpha}
$$

Finally, the heat rate limit becomes

$$
D<\frac{1}{2}\left(\frac{\dot{Q}_{\max }}{c V^{k}}\right)^{2} V^{2} S C_{D}
$$

In these expressions, $C_{D}$ and $L / D$ are computed using the angle of attack value from the nominal profile. Three drag boundary curves are defined by separately treating the inequalities of Eqs. (12-14) as equalities. A composite maximum drag profile is constructed by taking the minimum of the three drag boundary values at each energy value. This profile is $D_{\max }$ in Fig. 2. Using this method, the maximum drag profile will change slope discontinuosly at points when a different constraint becomes active. In order for the maximum drag profile to be flyable, cubic segments are applied at the corners to give a smooth transition from one constraint curve to another. Cubic segments are not able to match curvature as well as slope and value at 
both ends, so discontinuities are still seen in the second derivative of drag. Perfect tracking of such a drag profile would require a discontinuous change in bank angle at the points where cubic segments begin and end. Experimentation has shown that nearly perfect tracking can be achieved using a continous bank angle profile, provided that the cubic drag segment starts early enough. Even with cubic segments at the corners, the upper drag profile is not flyable in general because the initial and final drag conditions are not met. A simulation is performed in which bank angle is used to track the smoothed upper drag profile, starting from the initial entry conditions. The same feedback linearization (FBL) plus proportional-derivative (PD) controller as described in Saraf et $\mathrm{al}^{4}$ is used. The PD controller is critically damped. Due to rate saturation of $\sigma$, an overshoot in drag may occur if the natural frequency $\left(\omega_{n}\right)$ is too high. A search is performed for the value of $\omega_{n}$ that achieves the closest drag tracking without causing drag to overshoot. After $\omega_{n}$ has been found, a 5 deg bank maneuver is performed at the end to match the final drag condition. A search is performed to find the appropriate time to initiate the maneuver. The final product is called the flyable upper drag boundary and is labeled $D_{\max f}$ in Fig. 2.

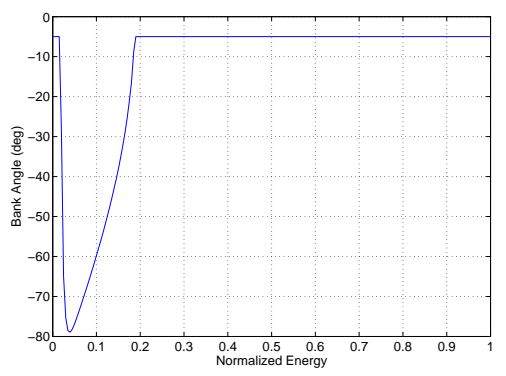

(a) commanded bank angle

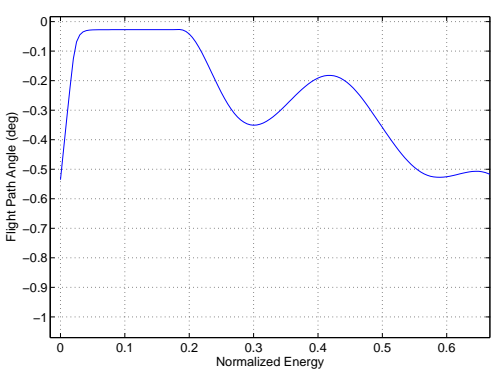

(b) flight path angle

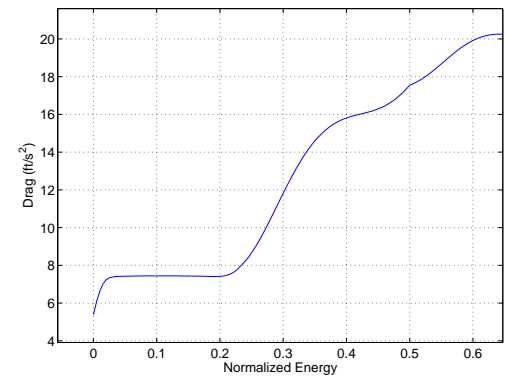

(c) resulting minimum drag profile

Figure 3. Example of gamma controller results

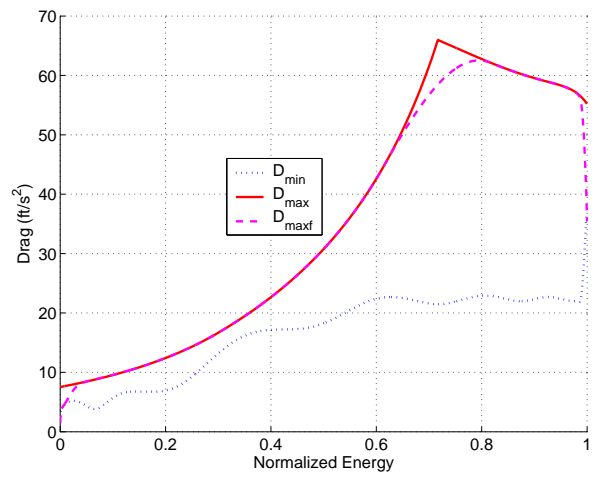

Figure 2. Drag boundary profiles

The lower drag boundary is usually determined by the equilibrium glide condition ${ }^{9}$ (i.e. the value of drag corresponding to $\gamma^{\prime}=0$ in Eq. 1 with $\sigma=0$ ). This condition however gives a "soft" constraint and the vehicle in a phugoid motion can periodically attain much lower drag values. We have limited our scope to trajectories that satisfy the condition $\gamma \leq 0.01$. This reduces the phugoid motion but does not negate it completely. If this constraint was not applied during the entire entry phase, it would travel the maximum distance. However, in general this would lead to skip outs. For the future it is planned to use an altitude controller instead of the $\gamma$ controller which would allow for a phugoid motion with a higher magnitude thus increasing the range while skip outs would be avoided.

To achieve the maximum distance without violating the imposed constraint of $\gamma \leq 0.01$, we use a simple feedback linearization based flight path controller to keep the flight path

angle close to zero during flight. If $\gamma_{r e f}(E)$ represents a reference flight path angle profile, then it is possible to track this profile using a first order error dynamics, given by

$$
\left(\gamma_{r e f}^{\prime}-\gamma^{\prime}\right)+K_{\gamma}\left(\gamma_{r e f}-\gamma\right)=0
$$

Replacing $\gamma^{\prime}$ from Eq. 1, we obtain

$$
\frac{L}{D} \cos \sigma=\frac{K_{\gamma} V}{D}\left(\gamma_{r e f}-\gamma\right)+\frac{1}{D}\left(g-\frac{V^{2}}{r}\right) \cos \gamma-\frac{2 \omega V}{D} \cos \psi \cos \phi
$$

Using $\mathrm{L} / \mathrm{D}$ values for angles of attack obtained from the angle of attack profile, it is possible to compute the bank angle $\sigma$ for all values of energy. The equations of motion are integrated from $\tilde{E}=0$ to 1 with the nominal angle of attack profile and bank angle from Eq. 16 as the inputs. 
Figure 3 shows a typical response achieved with the flight path angle controller. A model ${ }^{5}$ of a modified $\mathrm{X}-33$ has been used here to simulate an entry from orbit. Figure $3 \mathrm{~b}$ shows that $\gamma_{r e f}=-0.01$ deg is well tracked using bank angle in the early part of the entry. The resultant drag (Fig. 3c) is then used as the minimum allowable drag in the construction of the landing footprint. Note that the bank angle magnitude has been constrained to be $5 \mathrm{deg}$ or greater in the simulation (see Fig. 3a). This leaves some margin for maneuvering by a guidance algorithm, in case its target point lies near the maximum range boundary of the footprint. Also, it can been seen that after $\tilde{E}>0.19$ a $5 \mathrm{deg}$ bank command cannot balance forces in the vertical plane and the vehicle enters into a phugoid mode. For obtaining maximum range with a fixed bank angle, the phugoid motion cannot be avoided.

A constant bank maneuver is performed at the end of entry in order for the lower drag boundary to match the final drag condition, which is the drag value that corresponds to final velocity and altitude. This is the same as is done for the upper drag boundary, though the bank angle magnitude is taken to be 60 deg instead of $5 \mathrm{deg}$. See $D_{\min }$ in Fig. 2.

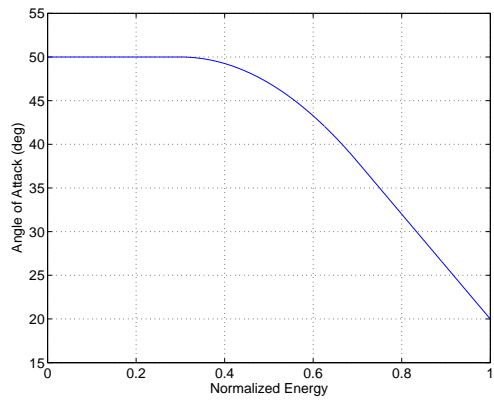

(a) common angle of attack profile

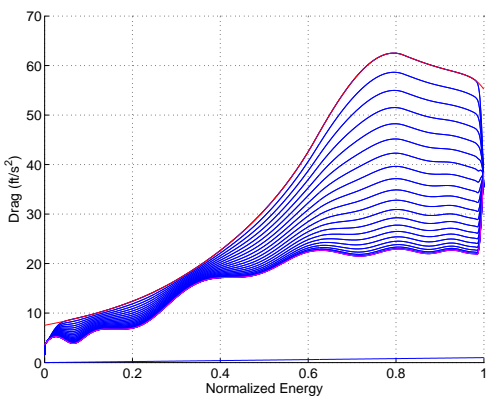

(b) interpolated drag profiles

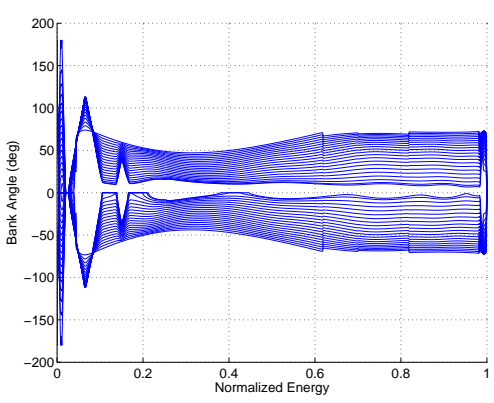

(c) bank angle profiles

Figure 4. Trajectory information for left and right side vertices of footprint with fixed alpha profile

The trajectories leading to the left and right boundaries of the landing footprint have drag profiles that lie in between the lower drag boundary and the flyable upper drag boundary. As previously stated, these trajectories achieve extreme crossrange by keeping a constant bank sign. Left-side trajectories bank left and right-side trajectories bank to the right. The task of finding flyable intermediate drag profiles is simplified by interpolating linearly between the upper and lower drag boundaries according to the relation

$$
D(E)=D_{\max f}(E)+c\left(D_{\min }(E)-D_{\max f}(E)\right)
$$

where $D_{\max f}$ is the flyable version of the upper drag boundary, $D_{\min }$ is the inherently flyable minimum drag profile, and $c$ is a constant between 0 and 1. Figure 4 shows the interpolated drag profiles of increasing $c$ value used to generate the left and right sides of the footprint shown in Fig. 1. The angle of attack profile that they have in common is shown in Fig. 4a, and their resulting bank angle profiles are shown in Fig. 4c. The interpolated drag profiles are not guaranteed to be flyable. However, it can be seen that the bank angle profiles in Fig. 4c show near-linear interpolation between the bank angle profiles that correspond to minimum drag and flyable maximum drag. As a consequence, the value, rate, and acceleration of the intermediate bank angle profiles are approximately bounded by the rate, value, and acceleration of two flyable trajectories.

The near edge of the landing footprint is created from $D_{\max f}$. The points that make up that edge differ in the timing of a single bank reversal. The two endpoints of the near edge are generated without a bank reversal and consequently are also extreme points on the sides of the landing footprint. If a bank reversal is added to one of these two trajectories, bank rate limits cause a drop in drag from $D_{\max f}$ starting when the reversal begins. This drop is modeled through integration of the equations of motion using an open-loop constant rate bank reversal command. After the reversal is over the same FBL plus PD drag tracking law is used to smoothly return the drag to $D_{\max f}$. This allows bank reversals to occur at values of $E$ where $D_{\max f}$ did not originally satisfy $|\sigma|=0$. Again, $\zeta=1$ is used along with the highest value of $\omega_{n}$ that does not result in an overshoot. As before, this value is found through a numerical search. The tracking simulation is terminated once the drag error and the drag slope error are both below certain tolerances. From this point until the final energy the ideal tracking response as determined by $\zeta$ and $\omega_{n}$ is assumed. If the drag error is not within tolerance when the final energy is reached, an open-loop bank maneuver is used to achieve this in 
the same way as was done for the no-reversal trajectories. Figure 5 shows the drag and bank angle profiles associated with one of the near-edge trajectories.

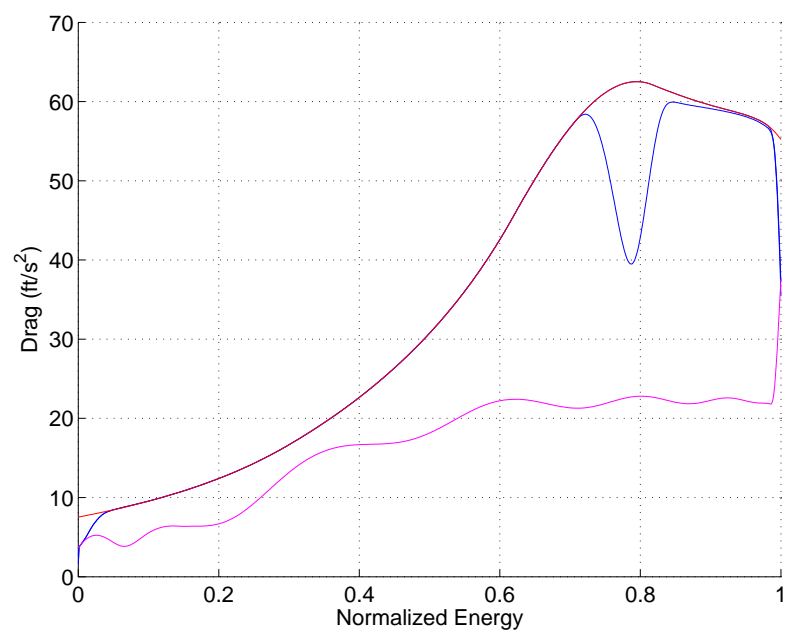

(a) drag

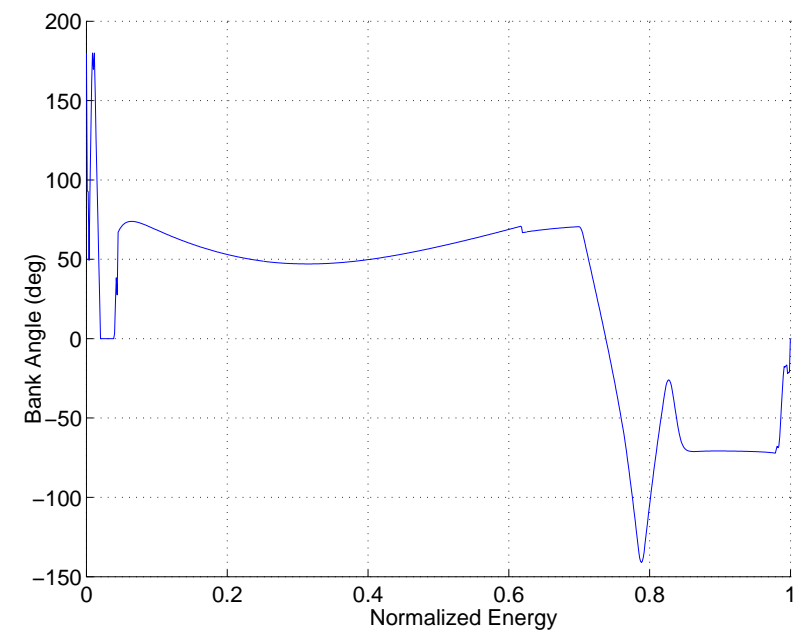

(b) bank angle

Figure 5. Minimum range trajectory with a bank reversal

As can be seen in Fig. 5a, drag drops significantly after a bank reversal. This is because lift is also maximum when flying $D_{\max f}$. During the bank reversal this high lift vector is rotated through the vertical position causing a rise in flight path angle and a large drop in drag. As seen in Fig. 1, the near edge of the landing footprint bows inward as a consequence of the extra range that results from drag dropping. The overall drag drop can be reduced considerably by reducing angle of attack during the bank reversal. Future work will examine adopting this technique for landing footprint generation. Another alternative would be to use bank reversals that occur in the first half of entry, when lift is low. This way the full crossrange would still be covered and the drop in drag would be minimized. There are practical entry guidance concerns, however, with using a single early bank reversal. If one were to generate another landing footprint just after the execution of the reversal, the trajectory endpoint would be right at the edge of the new footprint. This is not desirable until the vehicle is close to its target, ${ }^{4}$ since the accumulated effect of modeling errors could place the desired endpoint outside of the actual vehicle's capabilities.

The far edge of the landing footprint could be created in the same way as the near edge, using $D_{\min }$ instead of $D_{\max f}$. Since $5 \mathrm{deg}$ is taken as the minimum magnitude of $\sigma$, there is room for bank reversals. Observations indicate that the far edge is nearly straight, so the algorithm currently skips its computation and assumes it to be a straight line.

Though the method that has been presented in this section may seem extensive, total footprint computation time is less than one second on an Intel Pentium 4 2.66Ghz desktop computer running a non-optimized debugging build of the $\mathrm{C}$ implementation of the algorithm. Footprint computation time is equally as fast using the method described in the following section.

\section{B. Footprint with varying angle of attack profile}

The angle of attack profile has a significant effect on the landing footprint. To illustrate the effect, the procedure presented in the previous section is used for the same entry condition but different angle of attack profiles as shown in Fig. 6. It can be seen by comparing Fig. 6 with Fig. 1 that the two additional lower angle of attack profiles shown in Fig. 6 have created landing footprints with much more range. Hence by lowering the angle of attack, significant range improvement is possible.

Vinh $^{1}$ showed that the optimum angle of attack for a vehicle to glide maximum range during entry is the one that maximizes L/D under conditions of equilibrium glide and no heat rate constraint. Optimal angle of attack profiles generated with the trajectory optimization software ASTOS ${ }^{10}$ under the same conditions also maximized L/D. Usually during the entry, a vehicle flies with an angle of attack greater than that for maximum L/D. Reducing the angle of attack in the early entry phase may cause the vehicle heating limits to be violated. Optimal solutions generated with ASTOS imposing a heat rate constraint show that in order 


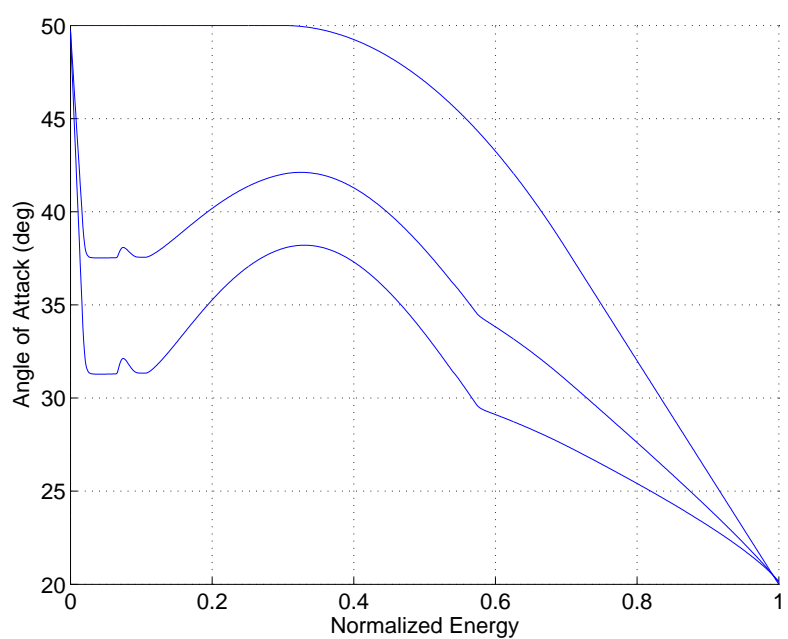

(a) angle of attack profiles

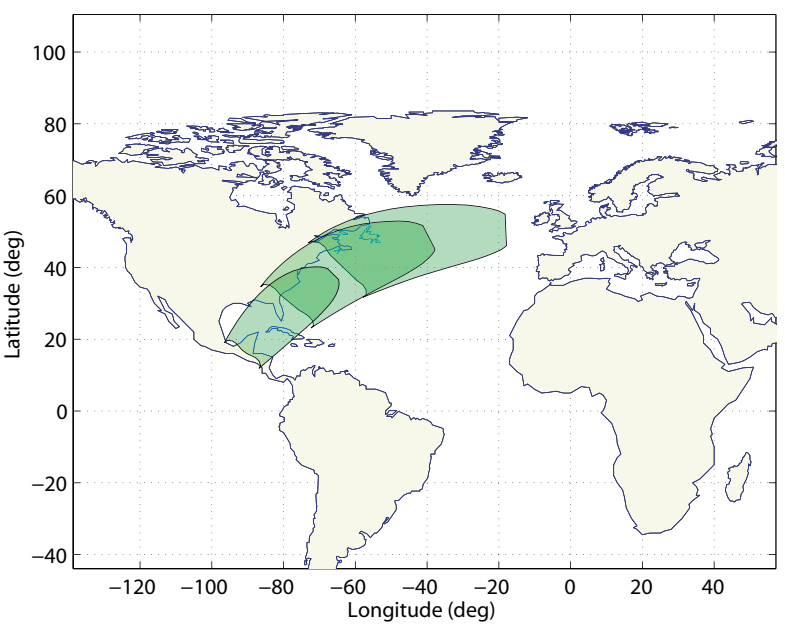

(b) landing footprints

Figure 6. Landing footprints generated with three angle of attack profiles

to fly higher range the angle of attack profile is to be lowered, but the angle of attack yielding maximum $\mathrm{L} / \mathrm{D}$ is only used when the heat rate constraint is not active. Hence to compute the maximum footprint of which a vehicle with freedom in its angle of attack profile is capable, an appropriate procedure for adjusting the angle of attack profile is required.

It can be seen from Eq. (7) that the heat rate limit is a function of density $\rho$ and velocity only. With $\rho$ being a function of $\mathrm{h}$ there exists a region in the altitude versus velocity plane where heating is within limits and this region is bounded from below by the maximum heat rate curve. If the vehicle flight path can be well controlled by bank angle modulation, it is advisable to fly at higher angles of attack to minimize heating. However, higher angles of attack also result in higher drag and that has an adverse effect if the vehicle needs to glide a large range. Optimal trajectories generated by ASTOS show the tendency to fly along the heat rate constraint until the vehicle's speed decreases significantly. Angle of attack is increased in these situations in order to avoid overheating.

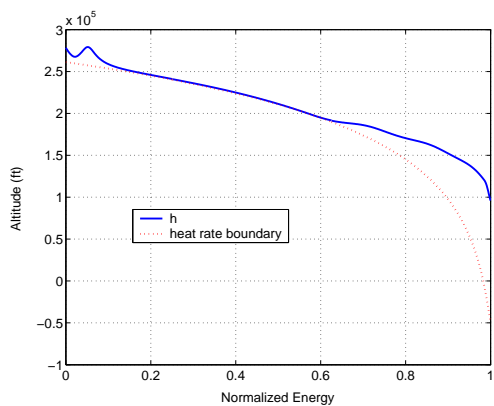

(a) altitude

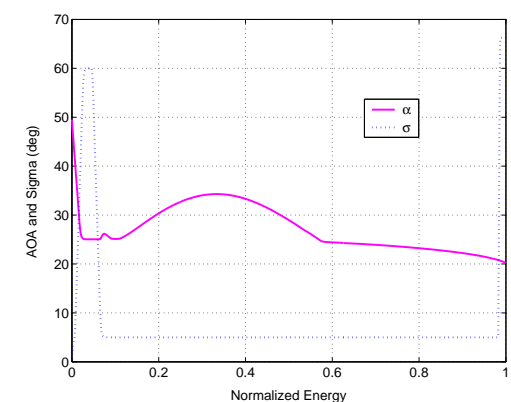

(b) control profiles

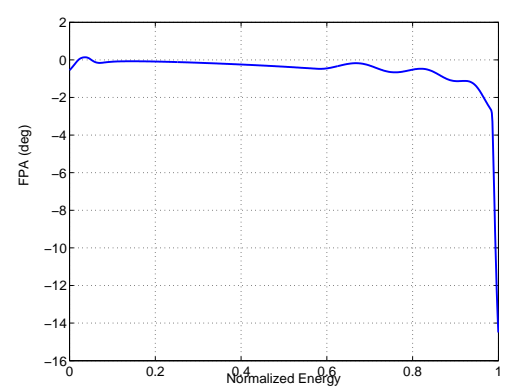

(c) flight path angle

Figure 7. Altitude controller results

Given the speed of current on-board processors direct multiple shooting or collocation methods such as used in ASTOS are not feasible for generating optimal angle of attack profiles in real-time. A method is presented that can generate near-optimal angle of attack profiles in a computationally efficient manner. This method uses an angle of attack based controller to track the heat rate curve in the altitude versus velocity plane. A feedback linearization based controller has been used for this purpose. The control law for $\alpha$ is designed to achieve the linear second-order dynamics for the error $h_{r e f}-h$

$$
h_{r e f}^{\prime \prime}-h^{\prime \prime}+2 \bar{\zeta} \bar{\omega}_{n}\left(h_{r e f}^{\prime}-h^{\prime}\right)+\bar{\omega}_{n}^{2}\left(h_{r e f}-h\right)=0
$$

where 


$$
\begin{aligned}
h_{\text {ref }} & =2 h_{s} \ln \left(\frac{\dot{q}_{\max }}{\sqrt{\rho_{0}} c V^{3.15}}\right) \\
h_{\text {ref }}^{\prime} & =6.3 h_{s} \frac{D+g \sin \gamma}{D V^{2}} \\
h_{\text {ref }}^{\prime \prime} & =6.3 h_{s}\left(\frac{D^{\prime}+g^{\prime} \sin \gamma+g \gamma^{\prime} \cos \gamma}{D V^{2}}-\frac{(D+g \sin \gamma) D^{\prime}}{D^{2} V^{2}}+\frac{(D+g \sin \gamma) 2 V^{\prime}}{D V^{3}}\right)
\end{aligned}
$$

The derivatives of the state variable $\mathrm{h}$ are given by

$$
\begin{aligned}
h^{\prime} & =-\frac{\sin \gamma}{D} \\
h^{\prime \prime} & =-\frac{\gamma^{\prime} \cos \gamma}{D}+\frac{\sin \gamma D^{\prime}}{D^{2}}
\end{aligned}
$$

The symbols $\bar{\zeta}$ and $\bar{\omega}$ represent the damping ratio and the natural frequency for the error dynamics in the energy domain and are control law parameters to be tuned. The value of $\bar{\zeta}$ is taken to be 1 since an overshoot is not desirable.

Substituting Eqs. (19) into Eq. (18) yields an implicit control law for the required $\alpha$ which is solved numerically. The result is then limited by

$$
\alpha_{\max L / D} \leq \alpha \leq 50 \mathrm{deg}
$$

The upper limit comes from the limitations of the test vehicle's aerodynamic database. The lower limit is the angle of attack that corresponds to maximum L/D. This is the desired angle of attack for maximum range trajectories. When the control law yields $\alpha \leq \alpha_{\max L / D}$, the vehicle flies at maximum L/D rather than continuing to fly on the heat rate constraint boundary. Since fast variations of the angle of attack may cause non-uniform heating over the vehicle this control is substantially rate limited. The bank angle is computed using the same controller as for landing footprints for fixed angle of attack.

Having generated the angle of attack profile for maximum range, it is possible to compute the enlarged landing footprint. The method first computes the minimum and the maximum drag profile and the altitude profiles corresponding to these drag profiles. It then interpolates simultaneously between these altitude profiles (Fig. 8c) and the angle of attack profiles (Fig. 8b) in order to obtain an interpolated drag profile (Fig. 8d). Drag is not interpolated directly since such a profile would likely violate the heat rate constraint. Altitude profiles do not pose this problem because they are guaranteed to remain above the critical altitude $h_{\text {ref }}$. The drag profiles that result from altitude interpolation will still stack on top of one another without intersection if the minimum alpha profile is always below the maximum alpha profile and the maximum altitude profile is always above the minimum altitude profile. Figure 8 shows the enlarged footprint for the same entry condition used for the previous landing footprints shown in this paper. Also shown in Fig. 8 is the optimal landing footprint for the same entry condition found using ASTOS. Since only side boundary results were computed using ASTOS, the ASTOS landing footprint is represented as curve in the figure.

The optimal footprint in Fig. 8 shows that our trajectory planner has room for improvement in both crossrange and downrange capabilities. Future work will examine possible improvements. It would be possible to use an angle of attack profile that remains at the maximum limit of the aerodynamic database for generating the upper drag boundary. Simulations with such an angle of attack profile have proven to enlarge the landing footprint toward the entry point but at the cost of crossrange for intermediate range trajectories. A better interpolation method could be developed in order to enable the landing footprint generator to make use of these extreme angle of attack profiles without losing performance at higher downranges.

Vinh $^{1}$ also shows that damping the vehicle's phugoid motion by $\alpha$ modulation increases range. The optimal solutions from ASTOS produce a similar result. As can be seen in Fig. 9b the angle of attack drops to a value less than the angle of attack that yields maximum L/D at the beginning of the flight. Figure 9f shows that this happens when the vehicle's altitude increases. Lower angle of attack directly results in lower lift and lower drag. Decreasing the lift decreases the vehicle's upward movement which causes the phugoid's magnitude to decrease while range is increased by the reduced drag. Since Vinh does not impose

9 of 14 


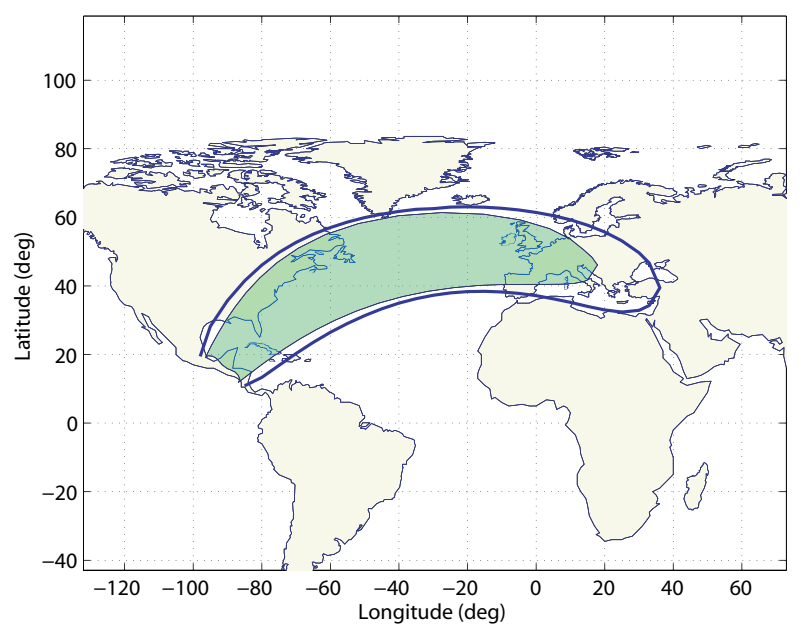

(a) landing footprint shown inside boundary of optimal landing footprint

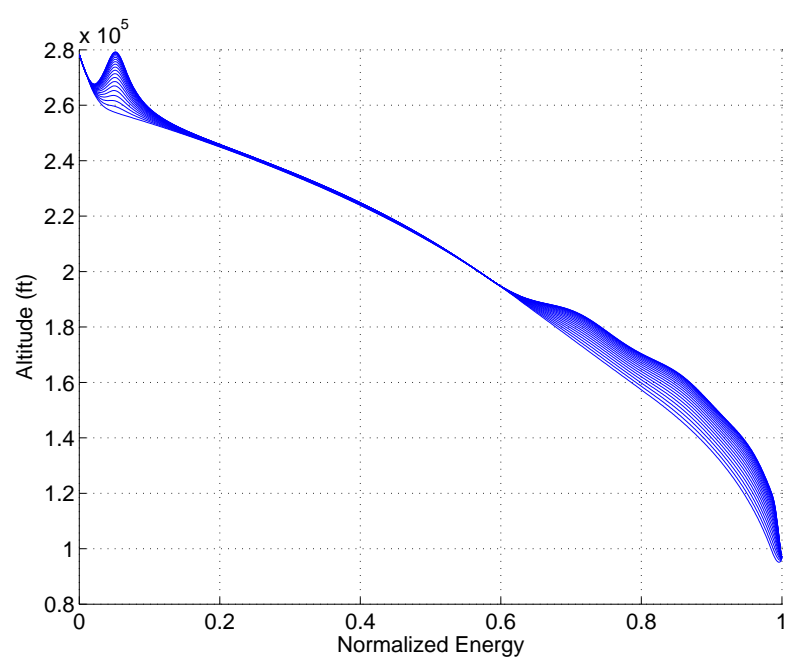

(c) altitude

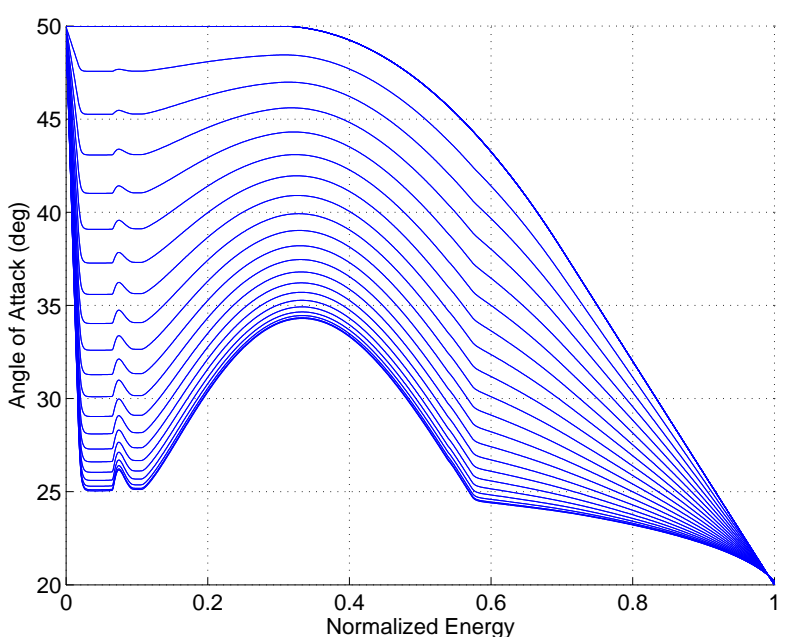

(b) angle of attack

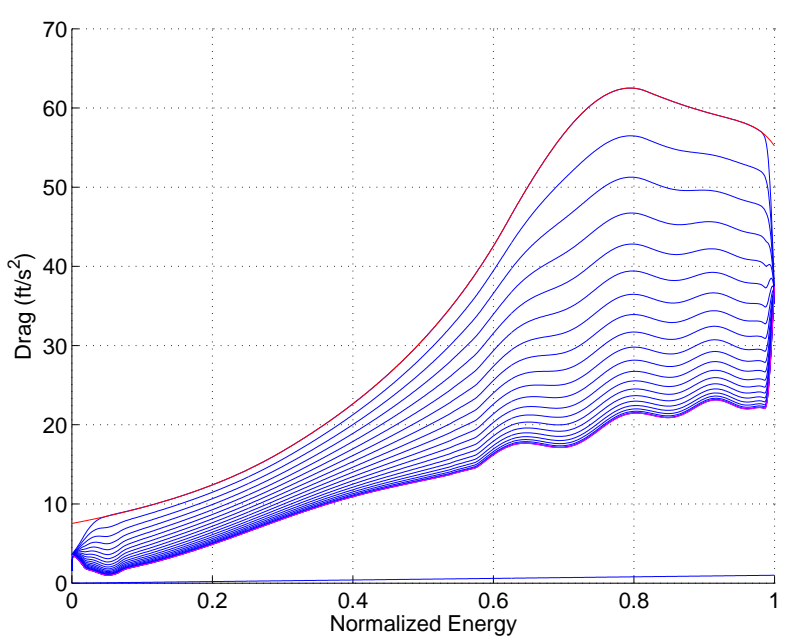

(d) drag

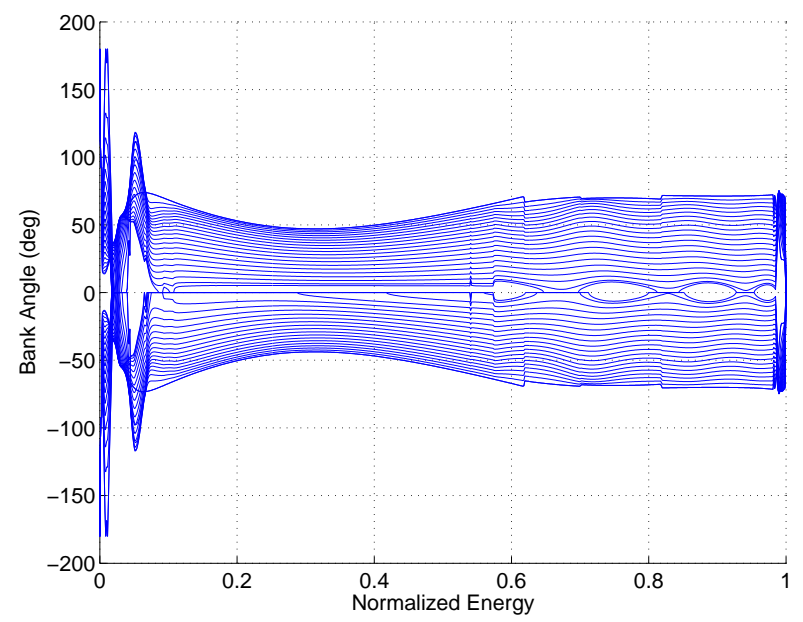

(e) bank angle

Figure 8. Landing footprint generated from varying angle of attack profile

10 of 14 


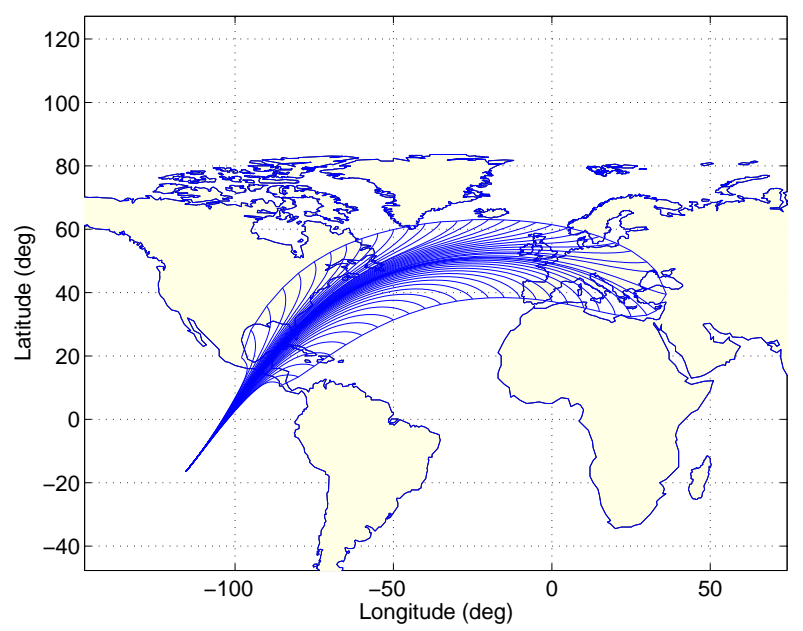

(a) landing footprint with ground tracks

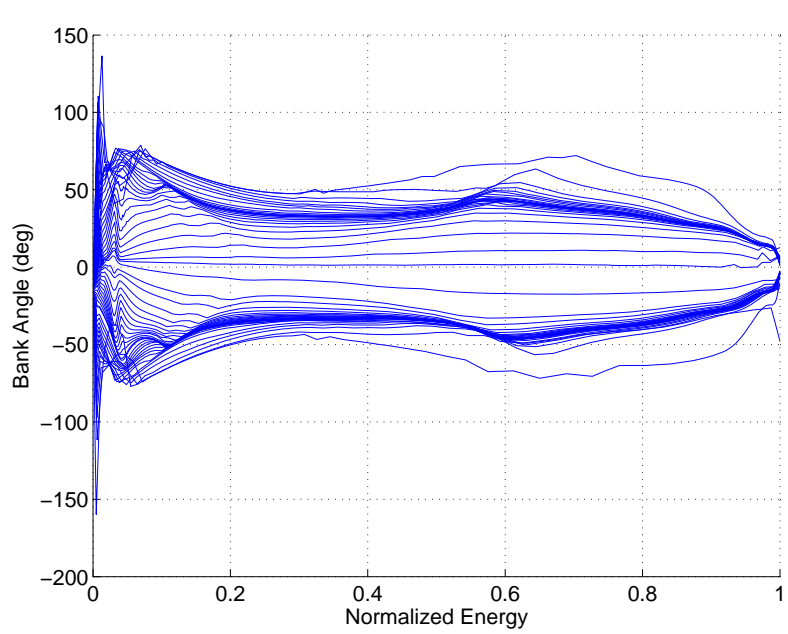

(c) bank angle profiles

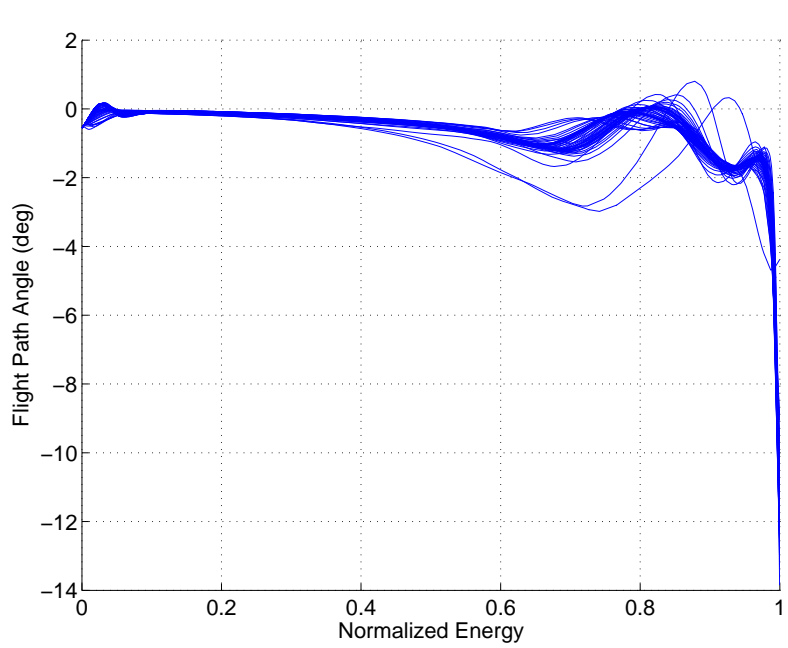

(e) flight path angle

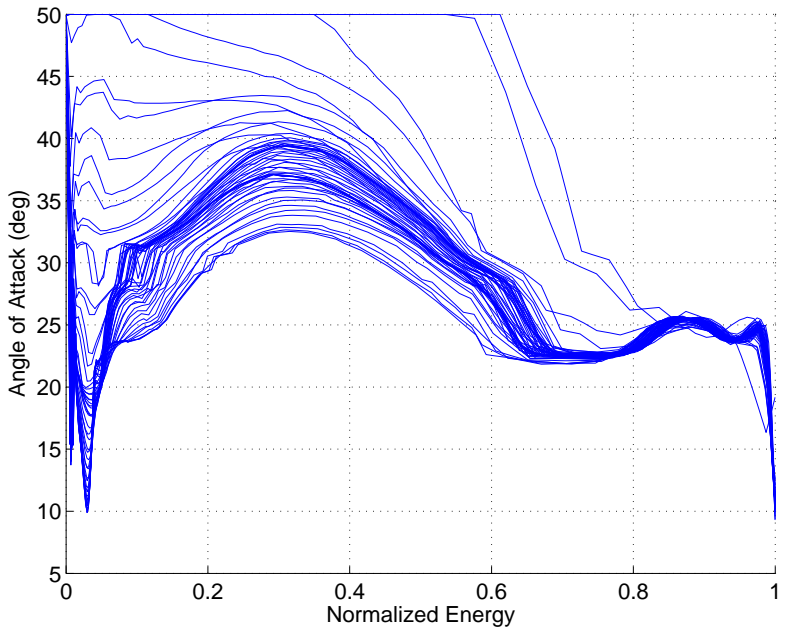

(b) alpha profiles

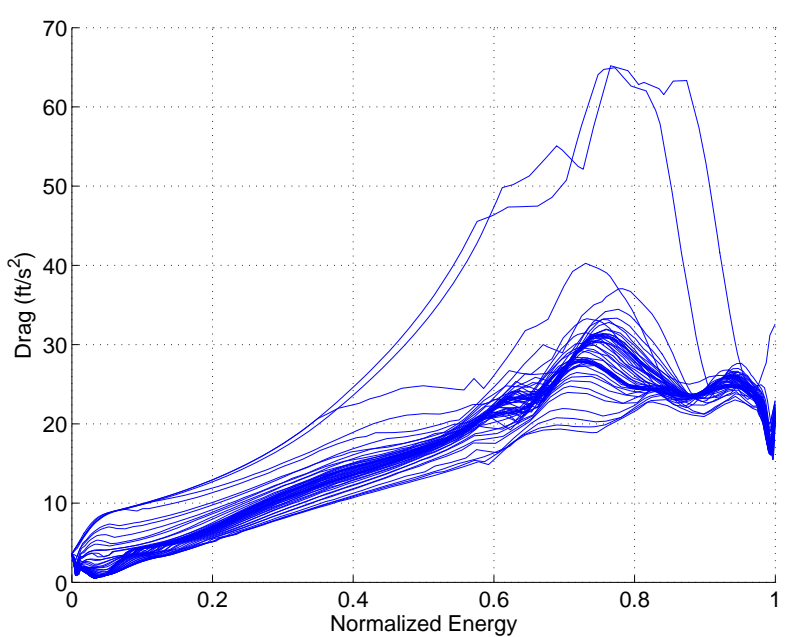

(d) drag profiles

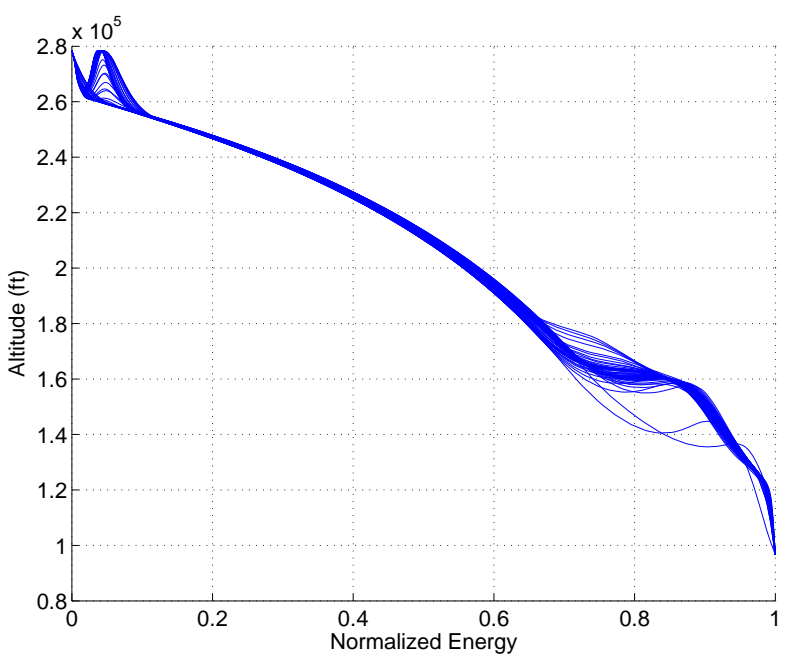

(f) altitude

Figure 9. Optimal landing footprint with trajectory information 
any path constraints such as maximum heat rate his solutions show phugoid motion over the whole flight. The optimal solution from ASTOS as well as the solution of the method presented herein fly along the heat rate constraint for a large part of the flight. Since flying along this constraint implies flying along a smooth monotonical decreasing altitude profile phugoid motion is precluded. As soon as the heat rate constraint becomes inactive a phugoid motion begins again. At this point the optimal solution decreases angle of attack in order to compensate. This ability has not been implemented into the method yet which explains why ASTOS achieves more downrange as can be seen in Fig. 8a.

\section{Results}

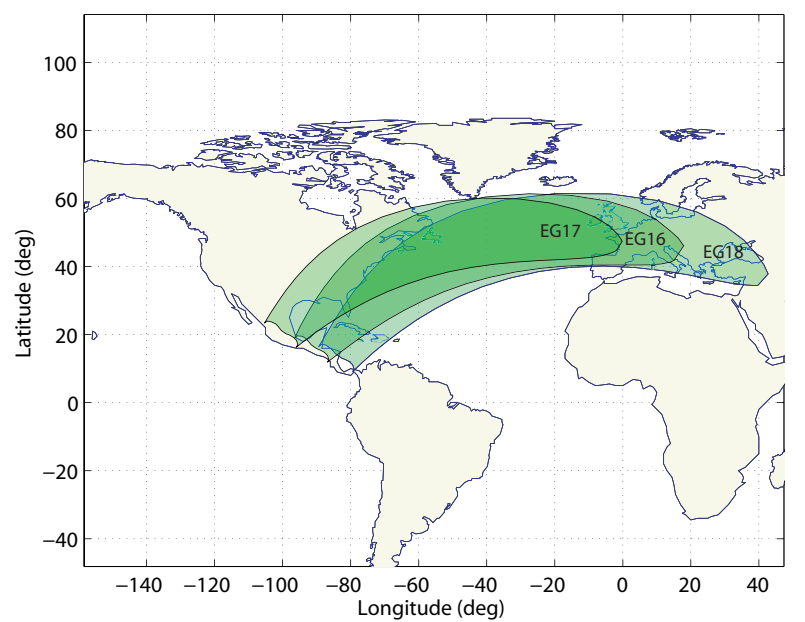

(a) landing footprints for EG16, EG17, EG18

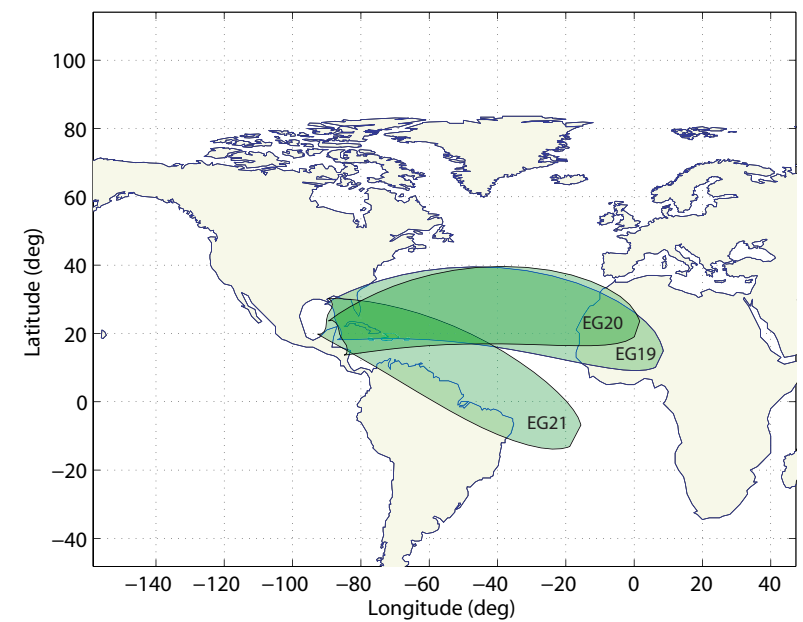

(b) landing footprints for EG19, EG20, EG21

Figure 10. Landing footprints with maximum range trajectory information for EG16 through EG21

A 3 degree of freedom model of the X-33 vehicle is used for the landing footprint computations. A dispersed entry condition for case EG16 of NASA's Advanced Guidance and Control (AGC) study ${ }^{5}$ has been used for all of the figures that precede this section. This trajectory corresponds to an early entry from the International Space Station orbit with an inclination of $51.6 \mathrm{deg}$. Figure 10 shows landing footprints created by the algorithm for cases EG16 to EG21 in the AGC study. Please refer to Ref. [5] for information regarding these cases. The control and state profiles of the trajectories used to create the EG17 to EG21 footprints are very similar to those for EG16.

As a demonstration of the robustness of the landing footprint algorithm, a sample landing footprint for one of the suborbital cases in the AGC study is presented in Fig. 11. It is noteworthy that the footprint shown in Fig. 11 was generated using the algorithm's unmodified configuration for orbital footprint computation. Though suborbital footprint research by the authors is in early stages, it is the goal of the authors to achieve a single algorithm that can handle footprint generation for all reasonable entry conditions.

\section{Conclusion}

A method for computing landing footprints in real-time has been developed which accounts for the Coriolis effect and respects heating, dynamic pressure, and normal acceleration constraints as well as flyability. The method uses a revised trajectory planner of an existing entry guidance algorithm. The landing footprints represent a set of endpoints of feasible trajectories generated by the planner. A comparison has been made between a landing footprint created by the algorithm and a landing footprint for the same initial condition created using a general purpose trajectory optimization software. The footprint made by the algorithm covers a large part of the optimal footprint, as expected since both longitudinal and lateral acceleration profiles are planned, angle of attack profiles are generated which are near-optimal for long range gliding, and the crossrange boundaries are achieved through constantly banking to the left or right. 
A landing footprint generated onboard at the time of an abort would aid in landing site selection. Although the execution time for computing footprints onboard a space vehicle has not been determined, the algorithm runs quickly on a desktop computer.

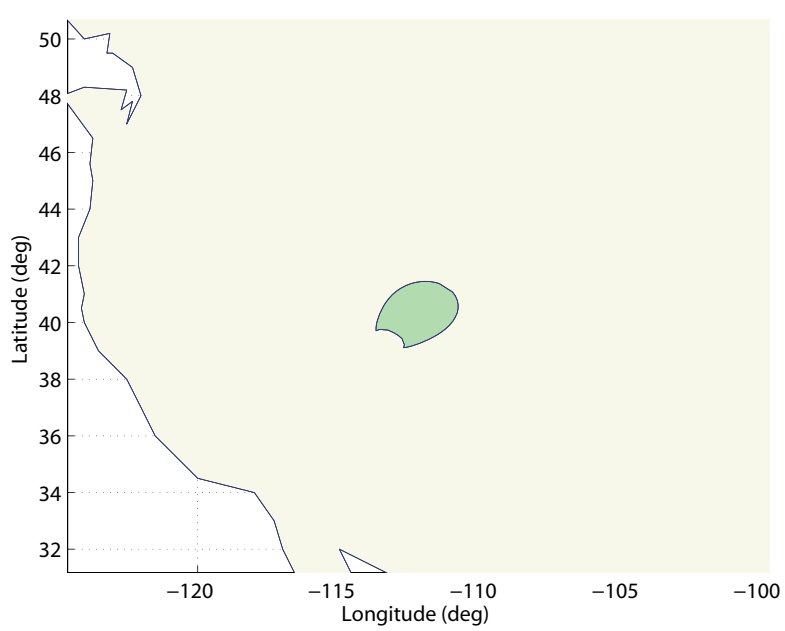

(a) landing footprint

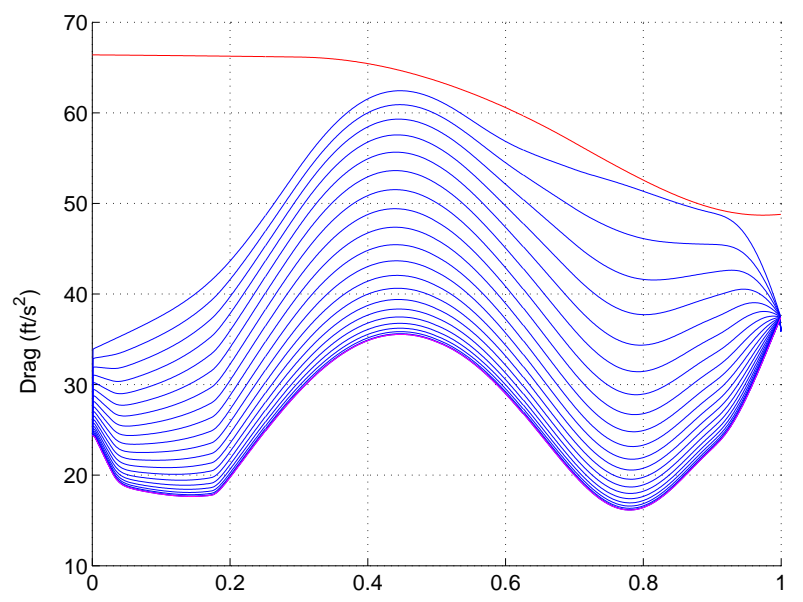

(c) drag profiles

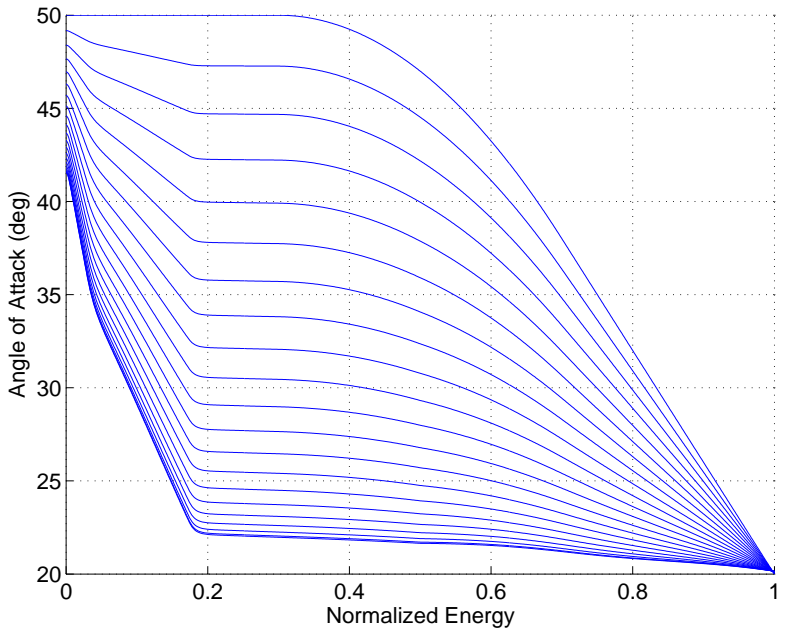

(b) alpha profiles

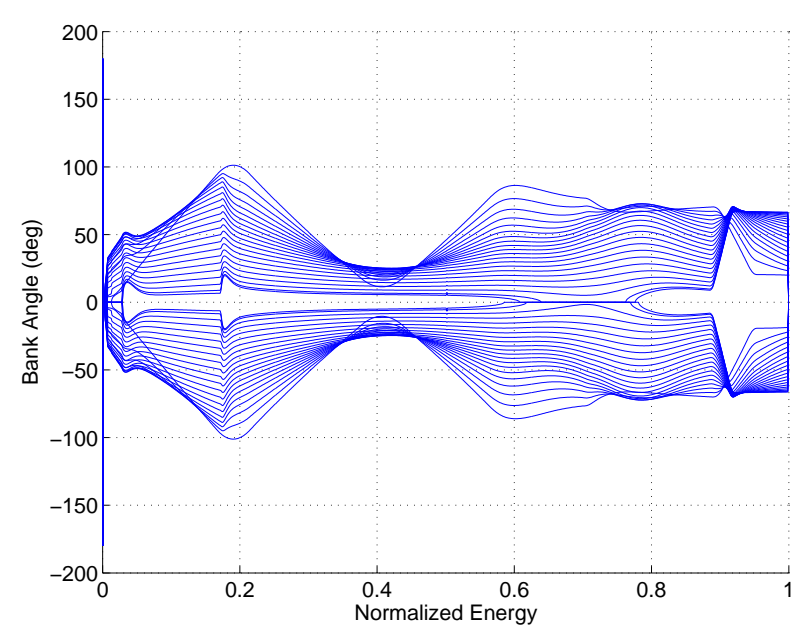

(d) bank angle profiles

Figure 11. Landing footprint for EG3 with trajectory information

\section{Acknowledgments}

This research was funded in part by Universal Space Lines, LLC and in part by Guided Systems Technologies, Inc. The academic license of ASTOS 5.0.0 and helpful technical support were provided free of charge by the Institute of Flight Mechanics and Control, University of Stuttgart, Germany.

\section{References}

${ }^{1}$ Vinh, N.X., Optimal Trajectories in Atmospheric Flight, Elsevier, New York, 1981.

${ }^{2}$ Ngo, A.D, and Doman, D.B., "Footprint Determination for Reusable Launch Vehicles Experiencing Control Effector Failures," AIAA Paper Number 2002-4775, Guidance, Navigation, and Control Conference, Monterey, Aug. 2002.

${ }^{3}$ Jackson, M.C., Straube, T.M., Fill, T.J., Barrows, T.M., and Nemeth, S., "Onboard Determination of Vehicle Glide Capability for the Shuttle Abort Flight Manager (SAFM)," IEEE Aerospace and Electronic Systems Society, Core Technologies for Space Systems Conference, Colorado Springs, Nov. 2002.

${ }^{4}$ Saraf, A., Leavitt, J.A., Chen, D.T. and Mease, K.D., "Design and Evaluation of an Acceleration Guidance Algorithm 
for Entry," Journal of Spacecraft and Rockets.

${ }^{5}$ Hanson, J. and Jones, R., "Advanced Guidance and Control Methods for Reusable Launch Vehicles: Test Results," AIAA Paper Number 2002-4561, Guidance, Navigation and Control Conference, Monterey, Aug. 2002.

${ }^{6}$ Stengel, R.F., "Optimal Guidance for the Space Shuttle Transition," Journal of Spacecraft and Rockets, Vol. 11, No. 3, 1974, pp. 173-179.

${ }^{7} \mathrm{Lu}$, P., "Entry Guidance and Trajectory Control for Reusable Launch Vehicle," Journal of Guidance, Control and Dynamics, Vol. 20, No. 1, 1997, pp. 143-149.

${ }^{8}$ Mease, K.D., Chen, D.T., Teufel, P., and Schönenberger, H., "Reduced-Order Entry Trajectory Planning for Acceleration Guidance," Journal of Guidance, Control, and Dynamics, Vol. 25, No. 2, 2002, pp. 257-266.

${ }^{9}$ Harpold, J.C., and Graves, C.A., Jr., "Shuttle Entry Guidance," Journal of Astronautical Sciences, Vol. 27, No. 3, 1979, pp. 239-268.

${ }^{10}$ ASTOS AeroSpace Trajectory Optimization Software, http://www.astos.de 